\title{
Influencia del incremento de masa en la variación de la energía mecánica y cantidad de movimiento lineal en un oscilador armónico simple horizontal
}

\author{
Influence of the increase in mass on the variation of mechanical energy and \\ momentum in a horizontal simple harmonic oscillator
}

Isidoro Enrique Tapia Segarra. ${ }^{1}$, Mayra Alejandra Pacheco Cunduri. ${ }^{2}$ \& Esteban Augusto Guevara Cabezas. $^{3}$

Recibido: 21-05-2020 /Aceptado: 13-06-2020 / Publicado: 03-07-2020

\begin{abstract}
.
DOI: https://doi.org/10.33262/concienciadigital.v3i3.1357

In the present study, the theory of Energy and Linear Moment is applied in the simple harmonic motion M.A.S. demonstrating that the increase in mass affects the conditions of a simple harmonic oscillator, depending on the position in which this disturbance occurs causes the mechanical energy to be reduced if this increase occurs in the equilibrium position, or be maintained in the event that the disturbance due to the increase in mass occurs at one end of the oscillation. With this application the two most important laws of the Physics study have been demonstrated, such as: The law of conservation of mechanical energy combined with the law of conservation of linear motion in inelastic shocks, in which the speed after impact It turns out to be the same since the bodies move with a joint speed.

Keywords: Mass Increase, Mechanical Energy, Simple Harmonic Oscillator, Amount of Movement.

1 Escuela Superior Politécnica de Chimborazo, Facultad de Informática y Electrónica, Riobamba, Ecuador, itapia@espoch.edu.ec

2 Escuela Superior Politécnica de Chimborazo, Facultad de Informática y Electrónica, Riobamba, Ecuador, mayra.pacheco@espoch.edu.ec

3 Escuela Superior Politécnica de Chimborazo, Facultad de Informática y Electrónica, Riobamba, Ecuador, esteban.guevara@espoch.edu.ec
\end{abstract}




\section{Resumen.}

En el presente estudio se aplica la teoría de la Energía y Momento Lineal en el Movimiento armónico simple M.A.S. demuestra que el incremento de masa afecta las condiciones de un oscilador armónico simple, dependiendo de la posición en la que se produce esta perturbación haciendo que la energía mecánica se reduzca en caso de que este incremento se produzca en la posición de equilibrio, o se mantenga en el caso de que la perturbación debido al incremento de masa se produzca en un extremo de la oscilación. Con esta aplicación se consigue demostrar las dos leyes más importantes del estudio de la Física como son: La ley de la conservación de la energía mecánica combinada con la ley de la conservación de movimiento lineal en choques inelásticos, en la que la velocidad después del impacto resulta ser la misma ya que los cuerpos se mueven con una velocidad conjunta.

Palabras claves: Incremento de masa, Energía mecánica, Oscilador Armónico Simple, Cantidad de Movimiento.

\section{Introducción.}

En el estudio de la física, resulta de especial importancia el análisis de movimientos oscilatorios y ondas debido a que están presentes en varios fenómenos propios de la naturaleza tales como sentarse en un columpio y hacerlo oscilar, los sismos, movimientos telúricos y sistemas que experimenten o impliquen vibraciones (de Mesa, 2005).

El movimiento oscilatorio se genera, debido a que un sistema determinado tiene la capacidad de volver exactamente a la configuración original o de equilibrio y debido a que existe una fuerza recuperadora que actúa en el mismo para que este lo haga. En el caso de un resorte, al separarlo de la posición de equilibrio, aparece una fuerza recuperadora (de tipo elástico) que tiende a devolverlo a esa posición de equilibrio. Es lo que sucede al soltarlo, solo que debido a que el sistema lleva una cierta velocidad, esta permite que se supera la posición de equilibrio inicial, y entonces nuevamente, actúa la fuerza de recuperación, pero esta vez en sentido opuesto y tiende a devolverlo nuevamente a la posición de equilibrio inicial. Este fenómeno se produce una y otra vez (Camacho, 2019).

El movimiento oscilatorio de un sistema mecánico constituye en la Ingeniería Mecánica y eléctrica, uno de los campos de estudio más importante y relevante. El oscilador armónico ndimensional o con $\mathrm{n}$ grados de libertad es un modelo bien conocido y elemental tanto en física como en matemáticas. Por ejemplo, aparece como el modelo lineal en el estudio de oscilaciones, que es un área de interés común en ambas disciplinas (Vélez, 2016). Existen varios tipos de sistemas oscilatorios, pero uno de estos sistemas que en varias ocasiones ha sido objeto de estudio, es el sistema conformado por una masa-resorte al cual conocemos como oscilador armónico, debido a las diferentes aristas que este puede presentar y sus múltiples aplicaciones (Domínguez, 2015). El movimiento oscilatorio de vaivén es especialmente sencillo en un caso que llamamos 
Movimiento Armónico Simple (MAS). Un sistema que se mueve así, también se dice que se comporta como un Oscilador Armónico Simple (OAS) (Donoso León, 2017). Entre los estudios más comúnmente realizados sobre este sistema, se encuentran aquellos relacionados con los efectos estáticos y dinámicos del mismo.

En el presente estudio se presenta la aplicación de la teoría de la Energía y momento Lineal en el Movimiento armónico simple M.A.S. (García Barneto \& Bolívar Raya, 2005), éste problema involucra un sistema masa resorte como una aplicación del movimiento armónico simple M.A.S., combinado con cantidad de movimiento lineal. El problema planteado consiste en analizar las distintas posiciones, velocidades y aceleraciones que tiene una masa unida a un resorte con un extremo fijo como una función del tiempo, si este sistema masa resorte es afectado o perturbado por un aumento de masa $\mathrm{m}$, esto hará que se modifique las condiciones de posición, velocidad y aceleración en las siguientes oscilaciones, de la misma manera se plantea determinar la variación de la energía mecánica del sistema que puede mantenerse constante o puede variar dependiendo de la posición en donde se produce el aumento de la masa que se pega a la masa $\mathrm{M}$ en dos posiciones diferentes con un choque inelástico, así como su período de oscilación que también se verá incrementado.

Para el respectivo estudio se utiliza la teoría del movimiento armónico simple combinado con la conservación de la cantidad de movimiento lineal con la aplicación de conceptos matemáticos, físicos como choques inelásticos y que despierta gran interés en los estudiantes de los primeros semestres de las carreras de Ingeniería (García Barneto \& Bolívar Raya, 2005).

\section{Metodología.}

Para el análisis cinemático y energético del sistema masa resorte se utiliza una maqueta artesanal que consta de una base metálica, en donde se monta el sistema masa resorte, una masa principal (M en kilogramos), la misma que va unida a un resorte de constante ( $\mathrm{K}$ en $\mathrm{N} / \mathrm{m}$ ), una vez puesto en marcha el movimiento se incrementa una masa ( $\mathrm{m}$ en kilogramos ) que quedará pegada a la masa principal M, dependiendo de la posición en donde se produzca éste incremento de masa se conservará o se reducirá la cantidad de movimiento lineal y la energía mecánica $\mathrm{E}$ siendo ésta la suma de la energía cinética Kc y la energía potencial elástica $U_{\mathrm{E}}$ (Sears \& Young, 2009). según la ecuación:

$$
\mathrm{E}=\mathrm{Kc}+\mathrm{U}_{\mathrm{E}}(\mathrm{J})(\text { Joule })
$$

Para analizar el movimiento se han considerado dos experimentos en condiciones de posición distintas, para lo cual se partirá de condiciones iniciales de posición Xo y velocidad en el eje $\mathrm{x}$ llamada Vox, se pone en marcha el sistema y se realiza un incremento de masa (m en $\mathrm{kg}$ ) produciendo una perturbación al sistema masa resorte en el instante en que la masa pasa la posición de equilibrio $\mathrm{x}=0$ con una velocidad positiva máxima $\mathrm{V}+$ en su recorrido hacia la derecha, en un segundo experimento esta perturbación se realizará cuando la masa $\mathrm{M}$ del sistema masa-resorte alcance la amplitud A positiva en donde tiene una velocidad nula $V=0$, en ésta 
posición se producirá el incremento de masa $\mathrm{m}$, en éste caso se pretende probar que la energía mecánica no se ve alterada pero si el período de oscilación (T en s), ya que el sistema se volverá más lento en su movimiento debido al incremento de masa ( $\mathrm{m}$ en $\mathrm{kg}$ ).

Figura 1. Oscilador armónico simple constituido por una masa y un resorte antes del incremento de masa.

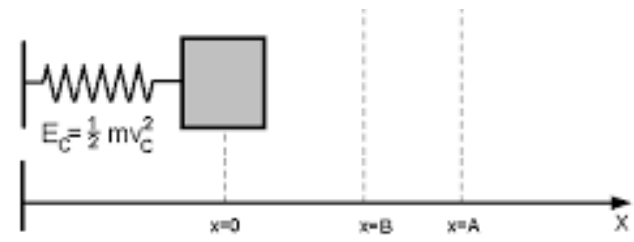

Fuente: Elaboración propia.

Primera condición de movimiento: Una vez que se ponga en marcha el oscilador armónico simple, se incrementará masa cuando el oscilador pase por la posición $\mathrm{x}=0$, en donde el oscilador tiene la velocidad máxima, está masa incrementada (m en $\mathrm{kg}$ ) quedará pegada a la masa del oscilador (M en kilogramos) (Serway \& Jewett, 2008), se desea probar que el incremento de masa del oscilador cambia su velocidad pasando de una velocidad inicial ( $\mathrm{u}$ en $\mathrm{m} / \mathrm{s})$ a una velocidad menor después del choque ( $\mathrm{v}$ en $\mathrm{m} / \mathrm{s}$ ) porque se produce una disminución de la cantidad de movimiento lineal $(\mathrm{P}$ en $\mathrm{kg} \cdot \mathrm{m} / \mathrm{s})$ después de producirse un choque inelástico, se determinará también su amplitud inicial (A1), la nueva amplitud (A2), debido a éste cambio de amplitud se cuantificará la Energía mecánica (E) que deberá pasar de una Energía inicial (E1 en J) a un nuevo valor de energía (E2 en J), se probará que de acuerdo a la teoría E2 debe ser menor que E1 es decir E2<E1, y que el período del oscilador pasará de un valor (T1 en s) a un período mayor (T2 en s), es decir T2>T1 (Gorri Ochoa et al., 2008).

Figura 2. Incremento de masa $m$ en la posición de equilibrio $x=0$ de la oscilación

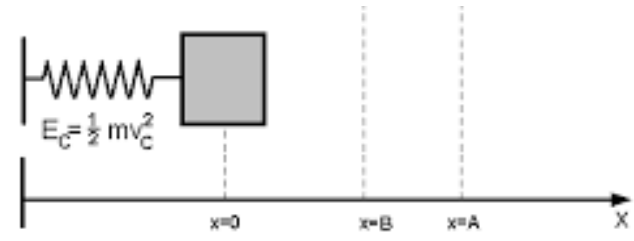

Fuente: Elaboración propia.

\section{Segunda condición de movimiento.}

Una vez que se ponga en marcha el oscilador armónico simple en la segunda condición de movimiento en la que se incrementará una masa ( $\mathrm{m}$ en $\mathrm{kg}$ ) cuando el oscilador pase por la posición $\mathrm{x}=\mathrm{A}$, en donde el oscilador tiene la velocidad nula, está masa incrementada quedará pegada a la masa del oscilador ( $\mathrm{M}$ en kilogramos) con la característica de ser un choque inelástico que hará que la masa del oscilador mantenga su amplitud A1=A2 después del choque (Sánchez, 2015), 
esto hará que cambie su período de oscilación pasando de un período (T1 en s) a un período mayor (T2 en s), es decir T2>T1 debido a que el sistema se vuelve más lento (Méndez \& Arrieta, 2007). Se desea probar también que el incremento de masa no afecta a la Energía mecánica (E en J), ésta se mantiene constante, es decir E1=E2 (Landau \& Lifshitz, 1970).

Figura 3. Incremento de masa $m$ en la posición extrema derecha $x=+A$ de la oscilación

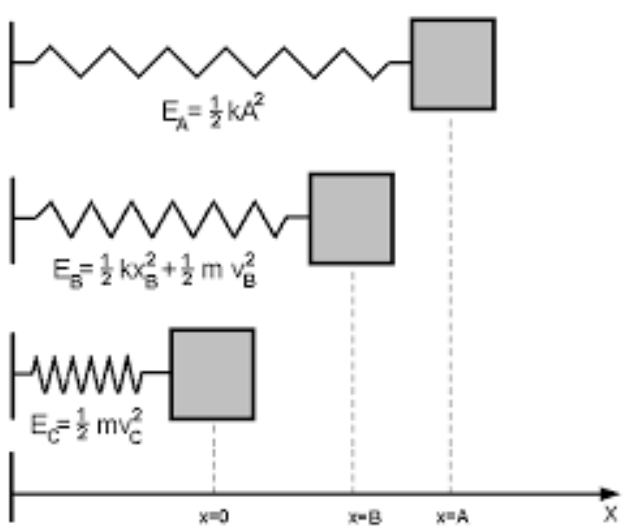

Fuente: Elaboración propia.

\section{Determinación del período $t$ y frecuencia angular $\mathbf{w}$}

En la figura 1 se muestra un oscilador armónico simple constituido por una masa $\mathrm{M}=10 \mathrm{~kg}$ y un resorte cuya constante elástica es $\mathrm{K}=100 \mathrm{~N} / \mathrm{m}$, con estos datos se determina el período de oscilación T1 mediante la ecuación 2:

$$
\begin{aligned}
& T 1=2 \pi * \sqrt{\frac{M}{K}}(\mathrm{~s}) \quad \text { ec. }(2) \\
& T 1=2 \pi * \sqrt{\frac{10}{100}}=1.99 \mathrm{~s} \\
& T 1=1.99 \mathrm{~s}
\end{aligned}
$$

La frecuencia angular $w$ se determina mediante la ecuación 3 y con ella también se determinará $w^{2}$ :

$$
\begin{aligned}
& w=\sqrt{\frac{K}{M}} \quad(\mathrm{rad} / \mathrm{s}) \\
& w=\sqrt{\frac{100}{10}}=3.16 \mathrm{rad} / \mathrm{s} \\
& w^{2}=10(\mathrm{rad} / \mathrm{s})^{2}
\end{aligned}
$$




\section{Determinación de la amplitud a1 y del ángulo de fase $\phi$}

La amplitud A1 viene determinada por la ecuación 4, en donde $(\mathrm{Xo}=0.18 \mathrm{~m})$ representa el desplazamiento inicial del oscilador, y (Vox $=1.00 \mathrm{~m} / \mathrm{s}$ ) representa la velocidad inicial positiva del oscilador:

$$
\begin{aligned}
& A 1=\sqrt{X o^{2}+\frac{V O X^{2}}{W^{2}}}(\mathrm{~m}) \quad \text { ec.(4) } \\
& A 1=\sqrt{0.18^{2}+\frac{1.0^{2}}{10}}=0.364(\mathrm{~m})
\end{aligned}
$$

El ángulo de fase $\boldsymbol{\Phi}$ se determina mediante la ecuación 5:

$$
\begin{aligned}
& \Phi=\arctan \left(-\frac{v o x}{w * X o}\right) \quad(\mathrm{rad}) \quad \text { ec.(5) } \\
& \Phi=\arctan \left(-\frac{1}{3.16 * 0.18}\right)=-1.054 \mathrm{rad}
\end{aligned}
$$

Determinación de las ecuaciones de posición, velocidad y aceleración del oscilador armónico simple.

$$
\begin{aligned}
& X(t)=A 1 * \cos (w * t+\Phi)(m)=0.364 * \cos (3.16 * t-1.054) \quad(\mathrm{m}) \\
& V(t)=-A 1 * w * \operatorname{sen}(w * t+\Phi)\left(\frac{m}{s}\right)=-0.364 * 3.16 * \operatorname{sen}(3.16 * \mathrm{t}-1.054) \quad(\mathrm{m} / \mathrm{s}) \quad \text { ec. }(7) \\
& a(t)=-A 1 * w^{2} * \cos (w * t+\Phi)\left(\frac{m}{s^{2}}\right)=-0.316 * 10 * \cos (3.16 * t-1.054) \quad\left(\frac{m}{s^{2}}\right) \text { ec. }(8)
\end{aligned}
$$

Comprobación de las ecuaciones de posición, velocidad y aceleración del oscilador armónico simple con los valores dados en las condiciones iniciales.

$$
\begin{gathered}
X(0)=A 1 * \cos (w * t+\Phi)(m)=0.364 * \cos (3.16 * 0-1.054)=0.179 \approx 0.18 \mathrm{~m} \quad \text { ec. }(6) \\
V(0)=-A 1 * w * \operatorname{sen}(w * t+\Phi)\left(\frac{m}{s}\right)=-0.364 * 3.16 * \operatorname{sen}(3.16 * 0-1.054)=1.00(\mathrm{~m} / \mathrm{s}) \quad \text { ec. }(7)
\end{gathered}
$$

Con lo que queda comprobado que las ecuaciones del movimiento están bien determinadas, porque los valores coinciden con las condiciones iniciales del problema planteado.

\section{Resultados obtenidos.}

Determinación de la velocidad máxima u1 del oscilador antes del incremento de masa m. 
La velocidad máxima del oscilador armónico simple se produce cuando la masa $\mathrm{M}$ alcanza la posición de equilibrio en $\mathrm{x}=0$, se tomará el valor positivo de la velocidad ( $\mathrm{u}$ en $\mathrm{m} / \mathrm{s}$ ), ésta velocidad es la medida antes del choque.

$$
\begin{aligned}
& u 1=w * \mathrm{~A} 1 \quad \text { ec. }(8) \\
& u 1=3.16(\mathrm{rad} / \mathrm{s}) * 0.364(\mathrm{~m})=+1.15 \mathrm{~m} / \mathrm{s}
\end{aligned}
$$

\section{Comprobación de la ecuación de la cantidad movimiento lineal para la primera condición de movimiento.}

Para choques inelásticos la cantidad de movimiento lineal antes del incremento de masa es igual a la cantidad de movimiento lineal después del incremento de masa, en donde $\mathrm{u} 2=0$ representa la velocidad de la masa incrementada, y V2 es la velocidad conjunta después del incremento de masa m, aplicando la ecuación de la cantidad de movimiento lineal se tiene:

$\mathrm{Po}=\mathrm{Pf}$

$\mathrm{M} * \mathrm{u} 1+\mathrm{m} * \mathrm{u} 2=(\mathrm{M}+\mathrm{m}) * \mathrm{~V} 2 \quad$ ec. $(9)$

Despejando V2, que representa la velocidad después del incremento de masa de la ecuación 9 se tiene la ecuación 9':

$$
\begin{aligned}
& V 2=\frac{M * u 1}{(M+m)}\left(\frac{m}{s}\right) \\
& V 2=\frac{10 * 1.15}{(10+1)}\left(\frac{m}{s}\right)=1.045\left(\frac{m}{s}\right)
\end{aligned}
$$

Se comprueba con los datos encontrados la ecuación 9 la conservación de la cantidad de movimiento lineal antes y después del incremento de masa es igual:

$$
10 * 1.15+0=(10+1) * 1.045
$$

\section{$11.5 \mathrm{~kg} . \mathrm{m} / \mathrm{s}=11.5 \mathrm{~kg} . \mathrm{m} / \mathrm{s}$}

\section{Determinación de la nueva amplitud del oscilador armónico simple para la primera condición de movimiento.}

La nueva amplitud máxima que alcanza el oscilador después de haber incrementado su masa es menor que la amplitud inicial A1:

$$
A 2=A 1 * \sqrt{\frac{M}{M+m}} \quad(m) \quad \text { ec. }(10)
$$




$$
A 2=0.364(\mathrm{~m}) * \sqrt{\frac{10}{10+1}}=0.347 \quad(\mathrm{~m})
$$

\section{$\mathrm{A} 2=0.347 \mathrm{~m}<\mathrm{A} 1=0.364 \mathrm{~m}$}

\section{Comprobación de la energía mecánica para la primera condición de movimiento.}

Para la primera condición de movimiento se desea comprobar que la energía mecánica no se conserva, ya que la amplitud A2 es menor que la amplitud A1:

La energía mecánica E1 antes del incremento de masa en el oscilador armónico simple es:

$$
\begin{aligned}
& E 1=\frac{1}{2} * K * A 1^{2} \\
& E 1=\frac{1}{2} * 100 * 0.364^{2}=6.62(\mathrm{~J})
\end{aligned}
$$

La energía mecánica E2 después del incremento de masa en el oscilador armónico simple es:

$$
\begin{aligned}
& E 2=\frac{1}{2} * K * A 2^{2} \\
& E 2=\frac{1}{2} * 100 * 0.347^{2}=6.01(\mathrm{~J})
\end{aligned}
$$

Por lo tanto, se puede establecer que existe una pérdida de energía mecánica porque la masa se desliza contra el bloque en movimiento durante el incremento de masa. E2<E1.

\section{Comprobación de la energía mecánica para la segunda condición de movimiento.}

En la segunda condición de movimiento cuando la masa se incrementa en el momento en que la velocidad que alcanza el oscilar es nula en el extremo positivo $\mathrm{X}=+\mathrm{A}$, aquí la cantidad de movimiento es nula antes y después del incremento de masa porque su velocidad es nula, y el incremento de masa no afecta a la energía mecánica. Es decir E1=E2, y la amplitud también es la misma debido a que $\mathrm{A} 1=\mathrm{A} 2$.

$$
\begin{aligned}
& \mathrm{E} 1=\mathrm{E} 2 \\
& E 1=\frac{1}{2} * K * A 1^{2} \quad(J) \quad \text { ec. }(11) \\
& E 2=\frac{1}{2} * K * A 2^{2} \quad(J) \quad \text { ec. }(12) \\
& E 1=E 2=\frac{1}{2} * 100 * 0.364^{2}=6.62 \quad(J)
\end{aligned}
$$




\section{Determinación del período de oscilación t2 después del incremento de masa m.}

El período de oscilación T2, es el tiempo que tarda el oscilador en dar una oscilación completa, este se verá incrementado en las dos condiciones de movimiento debido al incremento de masa, ya que el oscilador se mueve más lento, por lo tanto, el período de oscilación se incrementa:

$$
\begin{aligned}
& T 2=2 * \pi * \sqrt{\frac{M+m}{K}} \quad(s) \\
& T 2=2 * \pi * \sqrt{\frac{(10+1) k g * m}{100 N}}=2.08
\end{aligned}
$$

Con lo que queda determinado que $\mathrm{T} 2=2.08 \mathrm{~s}>\mathrm{T} 1=1.99 \mathrm{~s}$.

Gráficas de la posición, velocidad y aceleración del oscilador armónico simple en condiciones normales.

Figura 4. Gráficas en posición, velocidad y aceleración en función del tiempo
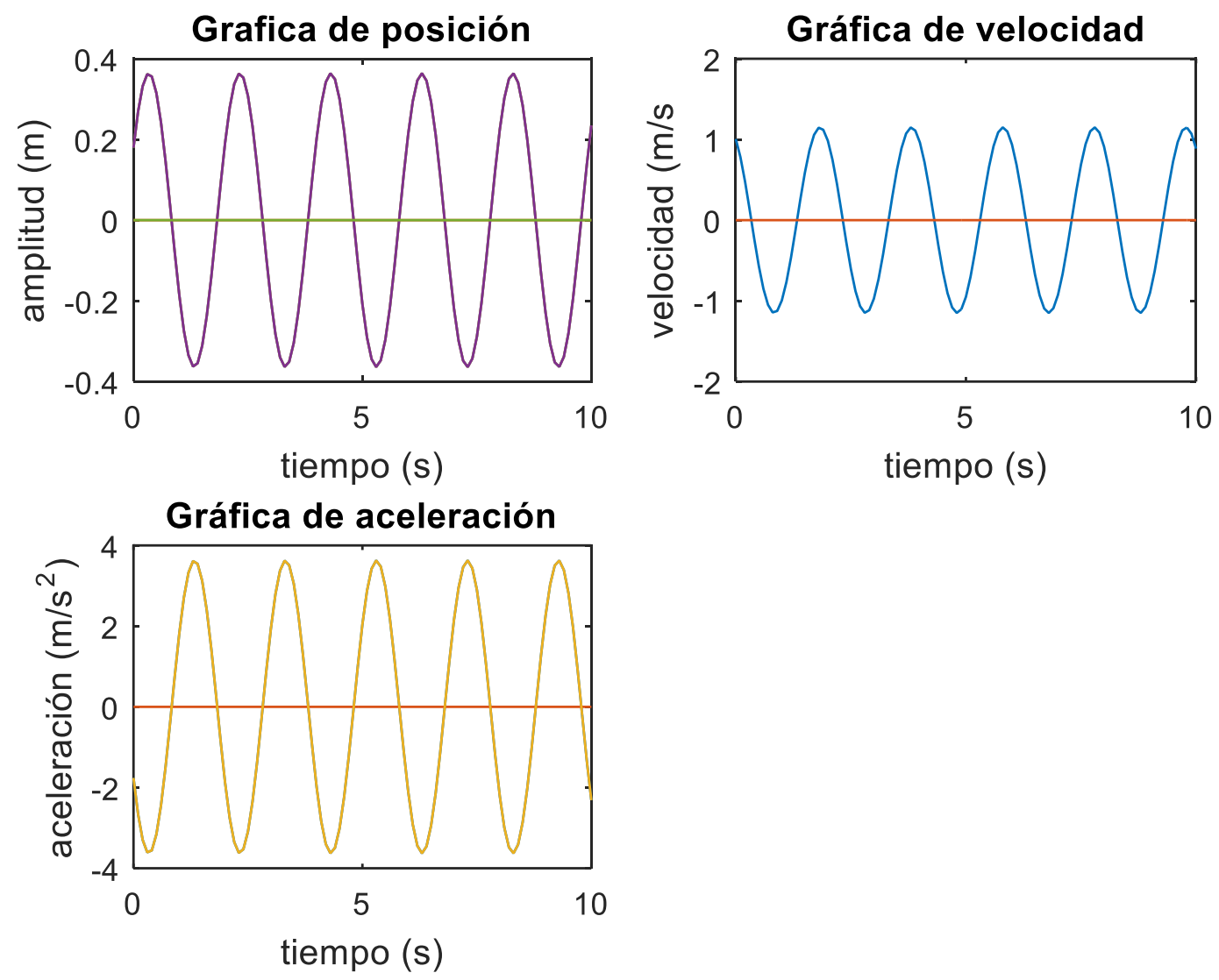

Fuente: Elaboración propia. 


\section{Conclusiones:}

- Como se ha podido comprobar las ecuaciones del movimiento armónico simple tienen la forma de una onda con un ángulo de desfase de $-1.054 \mathrm{rad}$, ya que la masa parte con condiciones iniciales $\mathrm{Xo}=0.18 \mathrm{~m}$, y con una velocidad inicial positiva $V o x=+1 \mathrm{~m} / \mathrm{s}$.

- En la posición de movimiento 1 el oscilador alcanza una velocidad máxima u1=+1.15 m/s cuando pasa por la posición $X=0$, en ésta posición se produce un incremento de masa $\mathrm{m}$ que produce una reducción de la velocidad a un valor V2=1.045 m/s. El período de oscilación cambia de $\mathrm{T} 1=1.99 \mathrm{~s}$ a $\mathrm{T} 2=2.08 \mathrm{~s}$. La amplitud también se reduce de A1=0.364 $\mathrm{m}$ a un valor $\mathrm{A} 2=0.347 \mathrm{~m}$ en las siguientes oscilaciones después del incremento de masa $\mathrm{m}$. Por lo tanto la energía mecánica cambia de $\mathrm{E} 1=6.62 \mathrm{~J}$ a $\mathrm{E} 2=6.02 \mathrm{~J}$, esto es debido al incremento de masa que hace que el oscilador no alcance la amplitud A1.

- En la posición de movimiento 2 el oscilador el oscilador alcanza el extremo superior derecho con una velocidad nula $\mathrm{u} 1=0 \mathrm{~m} / \mathrm{s}, \mathrm{V} 2=0 \mathrm{~m} / \mathrm{s}$, por lo tanto no hay energía cinética, siendo en éste punto solo energía potencial elástica máxima que es igual a la energía mecánica total $\mathrm{E}$, el incremento de masa no cambia esta energía acumulada en el resorte $\mathrm{E} 1=\mathrm{E} 2=6.62 \mathrm{~J}$, la amplitud se mantiene $\mathrm{A} 1=\mathrm{A} 2=0.634 \mathrm{~m}$, el período en las posteriores oscilaciones se incrementa de $\mathrm{T} 1=1.99 \mathrm{~s}$ a T2 $=2.08 \mathrm{~s}$.

- Se ha podido demostrar en éste experimento como afecta el incremento de masa en dos posiciones diferentes del oscilador, comprobando las dos leyes importantes en el estudio de la Física Clásica como son: la Ley de la conservación de la energía mecánica, conjuntamente con la Ley de la cantidad de movimiento lineal con la aplicación de choques inelásticos, que indica que para éste tipo de choques la cantidad de movimiento se conserva, a diferencia que la energía mecánica toma valores diferentes debido al intercambio de energía para la posición de movimiento 1, pero se conserva para la posición de movimiento 2, siendo la cantidad de movimiento lineal en ésta posición nula por encontrarse en ese punto el sistema con velocidad igual a cero.

\section{Referencias bibliográficas.}

Camacho, M. A. (2019). Geometría y Dinámica del oscilador armónico 2-dimensional. SAHUARUS. REVISTA ELECTRÓNICA DE MATEMÁTICAS. ISSN: 24485365, 4(1). https://sahuarus.mat.uson.mx/index.php/sahuarus/article/view/101

Vélez, N. (2016). Diseño de una unidad didáctica para el análisis del desplazamiento de un oscilador armónico mecánico. Propuesta basada en la implementación del computador para el registro gráfico del movimiento de un sistema masa resorte en tiempo real. [Trabajo de grado, Especialización en educación en tecnología, Universidad distrital Francisco José de Caldas]. Repositorio InstitucionalUniversidad distrital Francisco José de Caldas. http://hdl.handle.net/11349/5115

Domínguez, M. O. (2015). Análisis del sistema masa-resorte helicoidal. Ingenio Y Conciencia Boletín Científico De La Escuela Superior Ciudad Sahagún, 2(4). https://doi.org/10.29057/ess.v2i4.1380 
Donoso León, C. E. (2017). Análisis comparativo del uso del laboratorio virtual con el tradicional para el aprendizaje del movimiento armónico simple con los estudiantes de segundo semestre paralelos "a" y "b" de la carrera de Ingeniería Civil de la Universidad Nacional de Chimborazo [Trabajo de grado de maestría, Maestría en Ciencias de la Educación Aprendizaje de la Física, Universidad Nacional de Chimborazo, 2017]. Repositorio institucional-Universidad Nacional de Chimborazo. http://dspace.unach.edu.ec/handle/51000/3371

García Barneto, A., \& Bolívar Raya, J. P. (2005). Uso de simulaciones informáticas en la enseñanza de la física: movimientos armónico simple y ondulatorio. Enseñanza de las ciencias, (Extra).

Sears, Z., \& Young, F. (2009). Física universitaria ( 『12.】^a ed.). Pearson Education.

Serway, R. A., \& Jewett, J. W. (2008). Física para ciencias e ingeniería ( 『7.『^a ed.). Cengage Learning Editores, SA de CV.

Gorri Ochoa, J. A., Toribio Millán, E., \& Albareda Tiana, A. (2008). Oscilaciones y ondas ( 『2.』^a ed.). Edicions UPC. http://hdl.handle.net/2099.3/36729

Sánchez, L. P. (2015). MOVIMIENTO ARMÓNICO SIMPLE.

Méndez, M. E. M., \& Arrieta, J. (2007). ¿Cómo en el ejercicio de la práctica de modelación de un sistema de resortes se construyen modelos multilineales? Acta Latinoamericana de Matemática Educativa, 20, 444 - 449.

Landau, L. D., \& Lifshitz, E. M. (1970). Física teórica. Mecánica (『2.』^a ed.). Reverte S.A. 


\section{PARA CITAR EL ARTÍCULO INDEXADO.}

Tapia Segarra, I. E., Pacheco Cunduri, M. A., \& Guevara Cabezas, E. A. (2020). Influencia del incremento de masa en la variación de la energía mecánica y cantidad de movimiento lineal en un oscilador armónico simple horizontal. ConcienciaDigital, 3(3), 552-563. https://doi.org/10.33262/concienciadigital.v3i3.1357

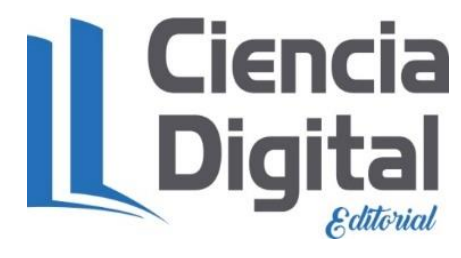

El artículo que se publica es de exclusiva responsabilidad de los autores y no necesariamente reflejan el pensamiento de la Revista Conciencia Digital.

El artículo queda en propiedad de la revista y, por tanto, su publicación parcial y/o total en otro medio tiene que ser autorizado por el director de la Revista Conciencia Digital.
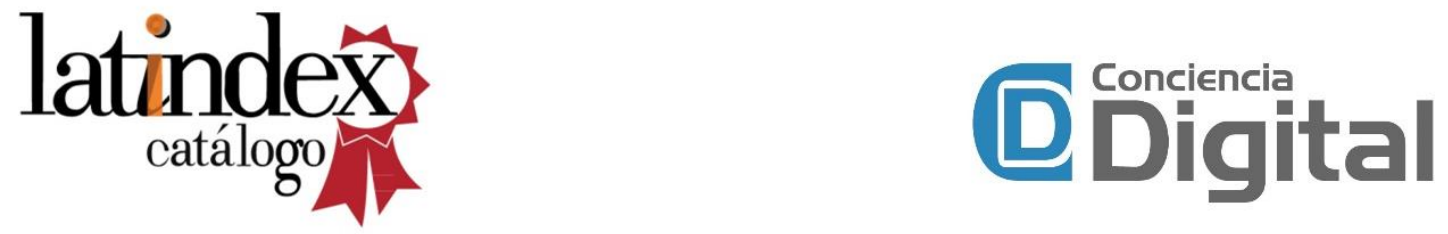